BNL-112397-2016-JA

\title{
Dependence of critical temperature in overdoped copper oxides on superfluid density
}

\author{
I. Bozovic, X. Hel, J. Wu \& A. T. Bollinger
}

Submitted to Nature

July 2016

\section{Condensed Matter Physics and Material Science Department \\ Brookhaven National Laboratory}

\author{
U.S. Department of Energy \\ USDOE Office of Science (SC), \\ Basic Energy Sciences (BES) (SC-22)
}

Notice: This manuscript has been authored by employees of Brookhaven Science Associates, LLC under Contract No. DE-SC0012704 with the U.S. Department of Energy. The publisher by accepting the manuscript for publication acknowledges that the United States Government retains a non-exclusive, paid-up, irrevocable, world-wide license to publish or reproduce the published form of this manuscript, or allow others to do so, for United States Government purposes. 


\section{DISCLAIMER}

This report was prepared as an account of work sponsored by an agency of the United States Government. Neither the United States Government nor any agency thereof, nor any of their employees, nor any of their contractors, subcontractors, or their employees, makes any warranty, express or implied, or assumes any legal liability or responsibility for the accuracy, completeness, or any third party's use or the results of such use of any information, apparatus, product, or process disclosed, or represents that its use would not infringe privately owned rights. Reference herein to any specific commercial product, process, or service by trade name, trademark, manufacturer, or otherwise, does not necessarily constitute or imply its endorsement, recommendation, or favoring by the United States Government or any agency thereof or its contractors or subcontractors. The views and opinions of authors expressed herein do not necessarily state or reflect those of the United States Government or any agency thereof. 


\title{
Dependence of critical temperature in overdoped copper oxides on
}

\section{superfluid density}

\author{
I. Božović, ${ }^{1,2, *}$, X. He ${ }^{1,2}$, J. Wu ${ }^{1}$ \& A. T. Bollinger ${ }^{1}$ \\ ${ }^{1}$ Brookhaven National Laboratory, Upton, New York 11973-5000, USA \\ ${ }^{2}$ Yale University, Applied Physics Department, New Haven CT 06520, USA
}

The physics of underdoped copper-oxide superconductors, including the pseudogap, spin and charge ordering, and their relation to superconductivity ${ }^{1-3}$, is intensely debated. The overdoped side is perceived as simpler, with strongly-correlated fermion physics evolving smoothly into the conventional Bardeen-Cooper-Schrieffer (BCS) behavior. Pioneering studies on a few overdoped samples ${ }^{4-11}$ indicated that the superfluid density was much smaller than expected, but this was attributed to pair-breaking, disorder, and phase separation. Here, we test this conjecture by studying how the magnetic penetration depth $\lambda$ and the phase stiffness $\rho_{s}$ depend on temperature and doping, scanning densely the entire overdoped side of the $\mathrm{La}_{2-x} \mathrm{Sr}_{x} \mathrm{CuO}_{4}$ (LSCO) phase diagram. We have measured the absolute values of $\lambda$ and $\rho_{s}$ to the accuracy of $\pm 1 \%$ in thousands of cuprate samples; the large statistics reveals clear trends and intrinsic properties. The films are quite homogeneous; variations in the critical temperature $\left(T_{c}\right)$ within a film are very small $(<1 \mathrm{~K})$. At every doping, $\rho_{s}(T)$ decreases linearly with temperature. The $T_{c}\left(\rho_{s o}\right)$ dependence is linear but with an offset, $\left(T_{c}-T_{0}\right) \propto \rho_{s 0}$ where $T_{0} \approx 7 \mathrm{~K}$, except very close to the origin where $T_{c}$ $\propto \vee \rho_{s}$. This scaling law defies the standard BCS description, posing a challenge to theory. 
Using atomic-layer-by-layer molecular-beam epitaxy (ALL-MBE) ${ }^{12-14}$, we synthesized singlecrystal films of LSCO, the simplest copper-oxide that we can dope all the way to nonsuperconducting metal. Since a single-layer LSCO sustains high-temperature superconductivity (HTS) with $T_{c}$ as high as in bulk samples ${ }^{14}$, the physics is quasi-two-dimensional (2D) and we focus on in-plane properties.

We used the mutual inductance technique ${ }^{9,10,15-17}$ (Fig. 1a-c and Extended Data Figs. 1 and 2), improved (see Methods) to resolve the absolute value of $\lambda$ with $\pm 1 \%$ accuracy. For this, it is critical to determine accurately the superconducting layer thickness, which we achieve by engineering the samples at the atomic-layer level as illustrated in Fig. 1d. The sharpness of the peak in $\operatorname{Im} V_{p}(T)$, or equivalently in $\operatorname{Im} M(T)$, where $V_{p}$ is the voltage on the pick-up coil and $M$ the mutual inductance, puts an upper bound of $\sim 0.1 \mathrm{~K}$ on the spread in $T_{c}$ (defined by the onset of Meissner effect) in this film, of $10 \times 10 \mathrm{~mm}^{2}$ area.

We have studied over 2,000 LSCO films in much detail (see Extended Data Figs. 3-8). The film thickness was varied from $d=0.66 \mathrm{~nm}$ (one-half the unit cell height) to over $100 \mathrm{~nm}$, and the composition across the entire phase diagram. This was decisive - copper-oxides are complex compounds, HTS has largely been a materials-science endeavor, and good statistics are essential.

In Fig. 2 we show our key experimental data extracted directly from the measured inductance and covering densely the entire overdoped LSCO region. Fig. 2a shows the doping dependence of $\lambda(T)$ for the 100 most homogeneous films, likely to represent the intrinsic LSCO properties. Figs. $2 \mathrm{~b}$ and $2 \mathrm{c}$ show the $2 \mathrm{D}$ superfluid phase stiffness $\rho_{s} \equiv A / \lambda^{2}$, which is directly proportional to the $2 \mathrm{D}$ superfluid density $n_{s}{ }^{2 D}=\rho_{s}\left(4 k_{B} m * / \hbar^{2}\right)$. Here $A=\hbar^{2} d / 4 \mu_{0} k_{B} e^{2}=3.55 \times 10^{-12} \mathrm{~m}^{2} \mathrm{~K}, k_{B}$ is the Boltzmann constant, $m^{*}$ the electron effective mass, $\hbar$ the reduced Planck constant, $\mu_{0}=$ 
$4 \pi \times 10^{-7} \mathrm{~N} / \mathrm{A}^{2}$ the vacuum permeability, and $e$ the electron charge. The $\rho_{s}(T)$ dependence is essentially linear, even in the heavily overdoped LSCO films (Fig. 2c). A crossover to a $T^{2}$ dependence occurs below some very low, sample-dependent temperature $T^{* *}$. Fig. $2 \mathrm{~d}$ shows the dependence of $T_{c}$ on $\rho_{s 0}$. (The subscript ' $O$ ' refers to the $T \rightarrow 0$ limit; this extrapolation is safe since our measurements extend down to $T=300 \mathrm{mK}$.) The $T_{c}\left(\rho_{s 0}\right)$ scaling in Fig. $2 \mathrm{~d}$ is largely linear, $T_{c}=T_{0}+\alpha \rho_{s 0}$, with $T_{0}=(7.0 \pm 0.1) \mathrm{K}$ and $\alpha=0.37 \pm 0.02$, except very close to the origin where the curve can fit well to $T_{c}=\gamma \sqrt{ } \rho_{s 0}$, with $\gamma=(4.2 \pm 0.5) \mathrm{K}^{1 / 2}$. The data (Extended Data Tables 1,2) are as accurate as depicted in the figure; the error bars are smaller than the marker size.

Our findings reinforce pioneering observations ${ }^{4-7}$ of the diminishing $\rho_{s 0}$ in a few overdoped Tl1201 samples, and subsequently also in overdoped $\mathrm{LSCO}^{8-11}$. We confirm this finding in thousands of LSCO films, and establish that $\rho_{s 0}$ decreases monotonously from the optimal doping until it vanishes and $T_{c}$ drops to zero.

The demise of $\rho_{s 0}$ with overdoping was originally attributed to spontaneous electronic phase separation ${ }^{4}$. However, quantum oscillations observed in overdoped Tl-2201 with $T_{c}=10 \mathrm{~K}$ indicate electronic homogeneity on the $100 \mathrm{~nm} \operatorname{scale}^{18,19}$. We show that LSCO films grown by ALLMBE are also intrinsically homogeneous, at every doping.

A nearly linear $\rho_{s}(T)$ dependence was observed ${ }^{20}$ by microwave technique in an overdoped Tl2201 crystal with $T_{c} \approx 25 \mathrm{~K}$ and very low $T^{* *} \approx 2 \mathrm{~K}$. We show that the same is true in LSCO films grown by ALL-MBE at every doping, with the slope independent on doping except very near the dome edge. 
However, our central result, the $T_{c}\left(\rho_{s 0}\right)$ scaling law shown in Fig. 2d, differs qualitatively from these early inferences. The well-known 'Uemura's Law', $T_{c} \propto n_{s} / m^{*}$, inferred from muon spin rotation $(\mu \mathrm{SR})$ measurements ${ }^{4}$, refers to the underdoped side. On the overdoped side, the scarce $\mu$ SR data were interpolated by a concave 'boomerang' shape ${ }^{5-7}$. In contrast, the $T_{c}\left(\rho_{s 0}\right)$ curve shown in Fig. $2 \mathrm{~d}$ is markedly convex. The same discrepancy is found with Homes' Law, $\rho_{s 0} \propto$ $\sigma_{d c} T_{c}$, (where $\sigma_{d c}$ is the normal-state conductivity measured near the $T_{c}$ onset) based on optics da$\operatorname{ta}^{23}$. According to Homes' Law and our measured $T_{c}$ and $\rho_{s 0}$ data, $\rho_{d c} \equiv 1 / \sigma_{d c}$ should increase with doping and diverge as $1 / \sqrt{ } \rho_{s 0}$ when $\rho_{s 0} \rightarrow 0$, because there $T_{c} \propto \sqrt{ } \rho_{s 0}$ (Extended Data Fig. 3). However, our measured $\rho_{d c}$ decreases monotonously (essentially linearly) with doping. These discrepancies may originate from the differences in the sample quality and homogeneity - cuprates are complex materials and call for advanced synthesis techniques — and in much larger error bars for $\rho_{s 0}$ values extracted from $\mu$ SR and optics data.

A study of high-quality, heavily underdoped YBCO crystals by microwave cavity-perturbation technique ${ }^{21,22}$ reported $\rho_{s}(T)$ curves linear down to $T^{* *} \approx 4 \mathrm{~K}$. The $T_{c} \propto \sqrt{ } \rho_{s 0}$ scaling was seen in a tiny range, $0.054<p<0.058$, near the superconductor-to-insulator transition and attributed to quantum critical fluctuations. At higher doping, the $T_{c}\left(\rho_{s 0}\right)$ dependence became linear with a clear offset, $T_{0} \approx 4-5 \mathrm{~K}$. This is reminiscent of our results shown in Fig. 2, but notably in a different compound, indicating that this behavior may be universal for the hole-doped copper-oxides, and on the underdoped side, suggesting underdoped/overdoped symmetry.

Our results, inferred from the raw data without any assumptions, challenge the prevalent creeds. In a clean BCS superconductor, $\rho_{s 0}$ should be equal to the total particle density. (In fact, Leggett's Theorem ${ }^{24,25}$ asserts that this is true as long as superfluidity conforms to the two-fluid sce- 
nario, and more generally for any single-species system that is translation- and time reversalinvariant.) Pair breaking by impurities and disorder can reduce $\rho_{s} 0$, but we doubt that our data can be quantitatively explained using the standard dirty d-wave BCS formalism, since in that case the Glover-Ferrell-Tinkham sum rule implies that Homes' Law should apply ${ }^{23,26}$, contrary to what we observe. Qualitatively, for $\rho_{s 0}$ to vanish because of disorder, the sample should get extremely dirty and one would observe a superconductor-to-insulator transition, while in fact Fig. 3b shows LSCO becoming more metallic.

The $\rho_{s}(T)$ dependence is expected to be linear only in a very clean d-wave BCS superconductor; disorder and pair breaking turn it quadratic $\mathrm{c}^{21,22,27}$ below $T^{* *}$. In Fig. $2 \mathrm{c}, T^{* *} \lesssim 2 \mathrm{~K}$, comparable to the high-quality $\mathrm{YBCO}$ crystals ${ }^{21,22}$ and corresponding within this formalism to a mean-freepath $l_{0} \gtrsim 4 \mathrm{~mm}$ and thus to the 'ultra-clean' limit. In Fig. 3a, we illustrate the effect of (deliberately added) disorder by comparing two samples with the same $T_{c}=38 \mathrm{~K}$, one slightly overdoped $(p=0.19)$ and clean, and the other optimally doped $(p=0.16)$ but with $0.5 \% \mathrm{Cu}$ replaced by Zn, a known pair-breaker. While this small amount of disorder does not affect $T_{c}$ much $\left(\Delta T_{c} \approx-3\right.$ $\mathrm{K})$, it has a dramatic effect on the $\rho_{s}(T)$ curve shape, which becomes parabolic below $20-25 \mathrm{~K}$. Evidently, we detect even a small amount of disorder and pair breaking.

The results we highlight here - the linear $\rho_{s}(T)$ dependence and the overall $T_{c}\left(\rho_{s 0}\right)$ scaling are robust and not sample-dependent, hence likely intrinsic. (On the contrary, $T^{* *}$ and $\rho_{d c}$ are indeed affected by disorder and other extrinsic factors; both can be modified by annealing the film in vacuum, oxygen, or ozone, thus modifying the concentration of oxygen vacancies, without much effect on $T_{c}$.) The extremely-dirty BCS picture is also inconsistent with observation of quantum oscillations and other experiments ${ }^{18,19,28}$. 
Homes' Law can be derived by a different line of reasoning ${ }^{28}$, assuming the 'Planckian' dissipation, $\tau=\hbar / k_{B} T$ - as strong as allowed by the uncertainty relation — which connects it naturally to another mysterious feature of HTS cuprates, the linear temperature dependence of resistivity. The latter is likely related to the linear $\rho_{s 0}(T)$ and $T_{c}\left(\rho_{s 0}\right)$ dependences we observe; as $T_{c}$ and $\rho_{s 0}$ decrease with overdoping, the $T$-linear term in resistivity decreases as well, and this may be the reason for deviation from the Homes' relation.

Another important inference is that $T_{c}$ appears essentially controlled by the superfluid density. It seems unlikely that the doping level $p$ is the primary factor controlling both $T_{c}$ and $\rho_{s}$, since $T_{c}$ strongly increases in LSCO under hydrostatic pressure or compressive epitaxial strain, which increases $\rho_{s 0}$ while keeping $p$ constant ${ }^{29}$. The underdoped/overdoped symmetry, even if only approximate, is another strong indication that $\rho_{s}$, rather than $p$, controls $T_{c}$. If $T_{c}$ is indeed essentially determined by the kinematics, this points to local pairing rather than to BCS. This notion is further supported by our extensive study of magnetoresistance in thousands of LSCO samples (I.B., J.W, A.T.B. \& X.H., unpublished), which together with the present one leads to the conclusion that the pair size is always smaller than their separation. However, this premise alone does not alleviate the paradoxes, since Leggett's Theorem ${ }^{23,24}$ remains valid for arbitrary interaction strength. Next, the fact that $T_{c}$ and $\rho_{s 0}$ are comparable points massive phase fluctuations ${ }^{17,30}$, questioning any mean-field description. Thus, our experimental findings presented here challenge the existing theories; however, the present accurate scaling may be a beacon. 


\section{References}

1. Lee, P. A., Nagaosa, N. \& Wen, X. G. Doping a Mott insulator: Physics of high-temperature superconductivity. Rev. Mod. Phys. 78, 17-85 (2006).

2. Zaanen, J., Chakravarty, S., Senthil, T., Anderson, P. W., Lee, P., Schmalian, J., Imada, M., Pines, D., Randeria, M., Varma, C., Vojta, M. \& Rice, M. Towards a complete theory of high $T_{c}$. Nature Phys. 2, 138-143 (2006).

3. Keimer, B., Kivelson, S. A., Norman, M. R., Uchida, S. \& Zaanen, J. From quantum matter to hightemperature superconductivity in copper oxides. Nature 518, 179-186 (2015).

4. Uemura, Y. J. et al. Universal correlations between $T_{c}$ and $n_{s} / m^{*}$ (carrier density over effective mass) in high- $T_{c}$ cuprate superconductors. Phys. Rev. Lett. 62, 2317-2320 (1989).

5. Uemura, Y. J. et al. Magnetic-field penetration depth in $\mathrm{Tl}_{2} \mathrm{Ba}_{2} \mathrm{CuO}_{6+\delta}$ in the overdoped regime. Nature 364, 605-607 (1993).

6. Niedermayer, C. et al. Muon spin rotation study of the correlation between $T_{c}$ and $n_{s} / m^{*}$ in overdoped $\mathrm{Tl}_{2} \mathrm{Ba}_{2} \mathrm{CuO}_{6+\delta}$. Phys. Rev. Lett. 71, 1764-1767 (1993).

7. Bernhard, C. et al. Magnetic penetration depth and condensate density of cuprate high- $T_{c}$ superconductors determined by muon-spin-rotation experiments. Phys. Rev. B 52, 10488-10498 (1995).

8. Panagopoulos, C. et al. Superfluid response in monolayer high- $T_{c}$ cuprates. Phys. Rev. B 67, 220502 (2003).

9. Locquet, J. P. et al. Variation of the in-plane penetration depth $\lambda_{a b}$ as a function of doping in $L_{2}$ ${ }_{x} \mathrm{Sr}_{x} \mathrm{CuO}_{4+\delta}$ thin films on $\mathrm{SrTiO}_{3}$ : implications for the overdoped state. Phys. Rev. B 54, 7481-7488 (1996).

10. Lemberger, T. R., et al. Superconductor-to-metal quantum phase transition in overdoped $\mathrm{La}_{2-}$ ${ }_{x} \mathrm{Sr}_{x} \mathrm{CuO}_{4}$. Phys. Rev. B 83, 140507 (2011).

11. Rourke, P. et al. Phase fluctuating superconductivity in overdoped $\mathrm{La}_{2-x} \mathrm{Sr}_{x} \mathrm{CuO}_{4}$. Nature Phys. 7, 455458 (2011).

12. Bozovic, I. Atomic-layer engineering of superconducting oxides: Yesterday, today, tomorrow. IEEE Trans. Appl. Supercond. 11, 2686-2695 (2001).

13. Gozar, A. et al. Interface superconductivity between a metal and a Mott insulator. Nature 455, 782785 (2008).

14. Logvenov, G., Gozar, A. \& Bozovic, I. High-temperature superconductivity in a single copper-oxygen plane. Science 326, 699-702 (2009).

15. Hebard, A. F. \& Fiory, A. T. Evidence for the Kosterlitz-Thouless transition in thin superconducting aluminum films. Phys. Rev. Lett. 44, 291-294 (1980).

16. Claassen, J. H., Reeves, M. E. \& Soulen, R. J. Jr. A contactless method for measurement of the critical current density and critical temperature of superconducting rings. Rev. Sci. Instrum. 62, 9961004 (1991). 
17. Clem, J. R. \& Coffey, M. W. Vortex dynamics in a type-II superconducting film and complex linearresponse functions. Phys. Rev. B 46, 14662-14674 (1992).

18. Vignolle B., et al. Quantum oscillations in an overdoped high- $T_{c}$ superconductor. Nature 455, 952-955 (2008).

19. Bangura, A. F. et al. Fermi surface and electronic homogeneity of the overdoped cuprate superconductor $\mathrm{Tl}_{2} \mathrm{Ba}_{2} \mathrm{CuO}_{6+\delta}$ as revealed by quantum oscillations. Phys. Rev. B 82, 140501(R) (2010).

20. Deepwell, D. et al. Microwave conductivity and superfluid density in strongly overdoped $\mathrm{Tl}_{2} \mathrm{Ba}_{2} \mathrm{CuO}_{6+\delta}$. Phys. Rev. B 88, 214509 (2013).

21. Hosseini, A. et al. Microwave spectroscopy of thermally excited quasiparticles in $\mathrm{YBa}_{2} \mathrm{Cu}_{3} \mathrm{O}_{6.99}$. Phys. Rev. B 60, 1349-1359 (1999).

22. Broun, D. M. et al. Superfluid density in a highly underdoped $\mathrm{YBa}_{2} \mathrm{Cu}_{3} \mathrm{O}_{6+y}$ superconductor. Phys. Rev. Lett. 99, 237003 (2007).

23. Homes, C. C. et al. A universal scaling relation in high-temperature superconductors. Nature $\mathbf{4 3 0}$, 539-541 (2004).

24. Leggett, A. Quantum Liquids (Oxford Univ. Press, 2006), chapters 3.3. and 3.4.

25. Leggett, A. On the superfluid fraction of an arbitrary many-body system at $T=0$. J. Stat. Phys. 93, 927-941 (1998).

26. Zaanen, J. Superconductivity: Why the temperature is high. Nature 430, 512-513 (2004).

27. Hirschfeld, P. J. \& Goldenfeld, N. Effect of strong scattering on the low-temperature penetration depth of a d-wave superconductor. Phys. Rev. B 48, 4219-4222 (1993).

28. Alldredge, J. W., et al. Evolution of the electronic excitation spectrum with strongly diminishing hole density in superconducting $\mathrm{Bi}_{2} \mathrm{Sr}_{2} \mathrm{CaCu}_{2} \mathrm{O}_{8+\delta}$. Nature Phys. 4, 319-326 (2008).

29. Bozovic, I., Logvenov, G., Belca, I., Narimbetov, B. \& Sveklo, I. Epitaxial strain and superconductivity in $\mathrm{La}_{2-x} \mathrm{Sr}_{x} \mathrm{CuO}_{4}$ thin films. Phys. Rev. Lett. 89, 107001 (2002).

30. Emery, V. \& Kivelson, S. A. Importance of phase fluctuations in superconductors with small superfluid density. Nature 374, 434-437 (1994).

\section{Acknowledgements}

A. Gozar, J. Zhang and J. Yoon contributed to developing the characterization techniques, at early stages of this work. R. Sundling developed the software for inversion of inductance data. We benefited also from the electrolyte-gating experiments and X-ray diffraction studies by X. Leng, and from numerical simulations by N. Bozovic. The research was done at BNL and was supported by the U.S. Department of Energy, Basic Energy Sciences, Materials Sciences and Engineering Division. X. H. is supported by the Gordon and Betty Moore Foundation. I.B. acknowledges valuable discussions with J. Zaanen, G. Deutscher, A. Leggett, P. Littlewood, C.-B. Eom, J. 
Mannhart, P. Coleman, R. Prozorov, D. van der Marel, A. McKenzie, V. Kogan, P. Armitage, JM. Triscone, P. Canfield, A. Chubukov, B. Halperin, Phillip Kim, T. Lemberger, M. V. Sadovskii, D. Pavuna, Z. Radović and M. Vanević.

\section{Author contributions}

I.B. conceived the project, synthesized films using ALL-MBE, measured inductance, analyzed the data, and wrote the text. X.H. synthesized films, performed AFM imaging, and measured inductance. A.T.B. fabricated the devices by lithography and performed inductance measurements in He3 system. J.W. performed the transport measurements.

*Correspondence and requests for materials should be addressed to I. Bozovic, e-mail: bozovic@bnl.gov. 

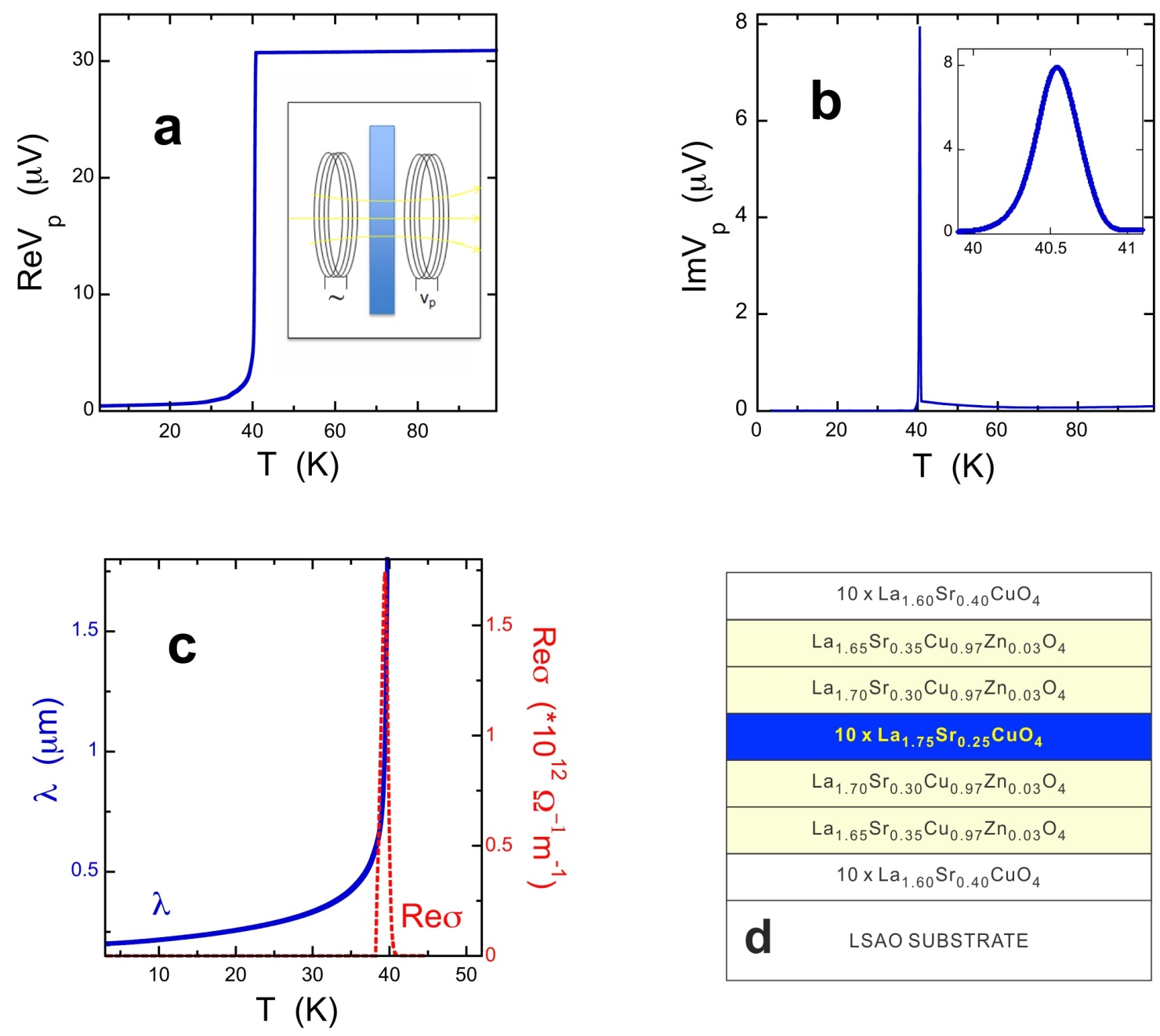

\begin{tabular}{|c|}
\hline $10 \times \mathrm{La}_{1.60} \mathrm{Sr}_{0.40} \mathrm{CuO}_{4}$ \\
\hline $\mathrm{La}_{1.65} \mathrm{Sr}_{0.35} \mathrm{Cu}_{0.97} \mathrm{Zn}_{0.03} \mathrm{O}_{4}$ \\
\hline $\mathrm{La}_{1.70} \mathrm{Sr}_{0.30} \mathrm{Cu}_{0.97} \mathrm{Zn}_{0.03} \mathrm{O}_{4}$ \\
\hline $10 \times \mathrm{La}_{1.75} \mathrm{Sr}_{0.25} \mathrm{CuO}_{4}$ \\
\hline $\mathrm{La}_{1.70} \mathrm{Sr}_{0.30} \mathrm{Cu}_{0.97} \mathrm{Zn}_{0.03} \mathrm{O}_{4}$ \\
\hline $\mathrm{La}_{1.65} \mathrm{Sr}_{0.35} \mathrm{Cu}_{0.97} \mathrm{Zn}_{0.03} \mathrm{O}_{4}$ \\
\hline $10 \times \mathrm{La}_{1.60} \mathrm{Sr}_{0.40} \mathrm{CuO}_{4}$ \\
\hline LSAO SUBSTRATE \\
\hline
\end{tabular}

Figure 1 Synthesis and characterization techniques. a, The in-phase component of $V_{p}$, the voltage across the pickup coil (proportional to the mutual inductance) showing diamagnetic screening (the Meissner effect) when the film becomes superconducting. The schematic of the experiment is shown in the inset. b, The imaginary part of $V_{p}$ shows that in this film, of $10 \times 10$ $\mathrm{mm}^{2}$ area, $T_{c}$ is homogeneous to better than $0.1 \mathrm{~K}$. c, The penetration depth, $\lambda$, and the real part of complex ac $(v=40 \mathrm{KHz})$ conductivity, Re $\sigma$, derived from the complex impedance. d, Schematic of a sample engineered for this study at the atomic-layer level. 

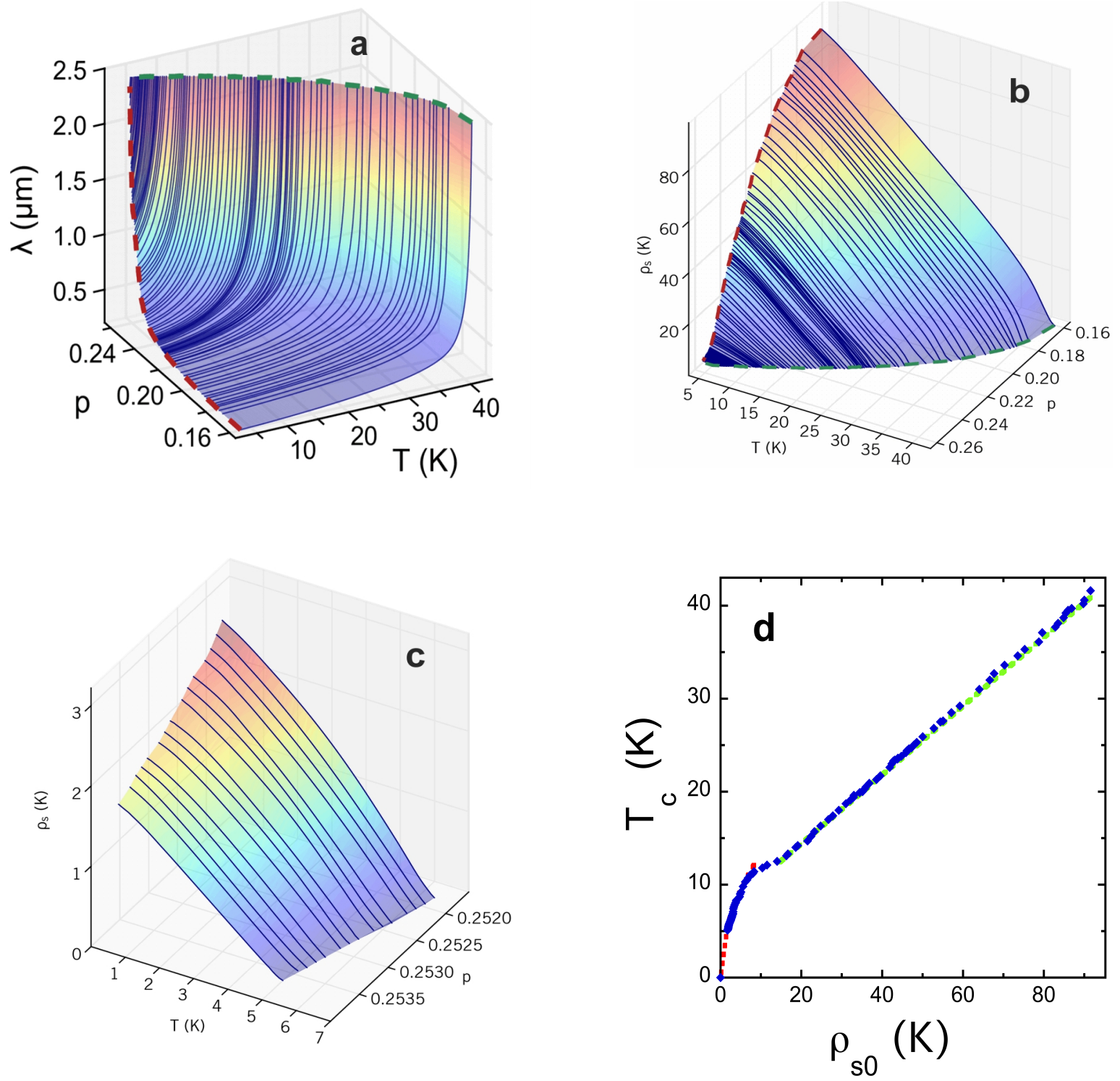

Figure 2 The evolution of superfluid with temperature and doping. a, The penetration depth $\lambda(T)$ measured in the 100 most homogeneous LSCO films, synthesized by ALL-MBE. b, The corresponding phase stiffness $\rho_{s}(T)$. c, The same, for the most overdoped samples, measured down to $T=0.3 \mathrm{~K}$. d, The dependence of $T_{c}$ on $\rho_{s 0} \equiv \rho_{s}(T \rightarrow 0)$. Experimental data: blue diamonds. Green dashed line: fit to $T_{c}=T_{0}+\alpha \rho_{s 0}$, with $\alpha=0.37 \pm 0.02$, for $\rho_{s 0}>15 \mathrm{~K}$. Red dashed line: fit to $T_{c}=\gamma \sqrt{ } \rho_{s 0}$, with $\gamma=(4.2 \pm 0.5) \mathrm{K}^{1 / 2}$, for $\rho_{s 0}<12 \mathrm{~K}$. 

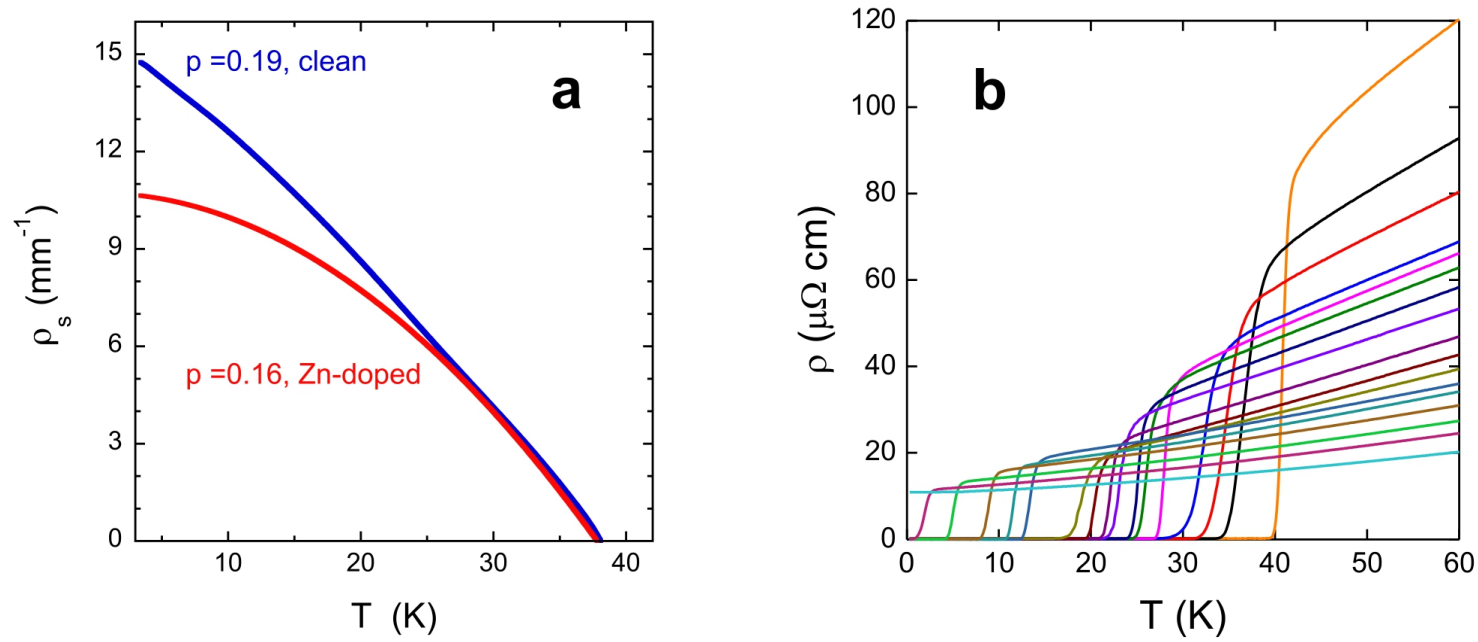

Figure 3 | Overdoped LSCO films synthesized by ALL-MBE are clean superconductors. (a) Red line: an LSCO film optimally doped $(p=0.16)$ but with $0.5 \%$ of $\mathrm{Cu}$ substituted by $\mathrm{Zn}$. Blue line: a clean LSCO film slightly overdoped $(p=0.19)$ to have the matching $T_{c} \approx 38 \mathrm{~K}$. In the clean film the $\rho_{S}(T)$ dependence is essentially linear, while in the 'dirty' one pair breaking makes it parabolic below about $25 \mathrm{~K}$. (b) Resistivity in LSCO films with different doping levels (see Methods), top to bottom: $p=0.172,0.205,0.211,0.217,0.220,0.224,0.228,0.230,0.233$, $0.237,0.242,0.245,0.248,0.251,0.254,0.258,0.295$. 


\section{METHODS}

\section{Atomic-layer-by-layer molecular beam epitaxy (ALL-MBE) synthesis}

In most HTS experiments so far, the main sources of uncertainty were the samples themselves. In complex materials like cuprates, some level of inhomogeneity is present in most samples due to extrinsic factors. Most HTS 'single crystals' in fact contain stacking faults and intergrowths of other cuprate phases and polytypes. Moreover, oxygen is volatile in cuprates, and hence bulk crystals are prone to gradients in the density of oxygen vacancies or interstitials. Irregular geometries of crystals and/or contacts cause some uncertainty in transport property measurements. In principle, one can alleviate the above problems by working with very thin single-crystal films; however, most HTS films are granular and contain both secondary phase precipitates and pinholes. This calls for some advanced materials science - as well as for large sample sets, with sufficient statistics to clearly discern intrinsic behavior.

For film synthesis, we use a custom atomic-layer-by-layer molecular beam epitaxy (ALL-MBE) system $^{12}$. It is equipped with 16 metal sources (either K-cells or rod-fed electron-beam sources), a pure ozone source, and 16-channel real-time rate monitoring system based on atomic absorption spectroscopy. It also contains a dual-deflection reflection high-energy electron diffraction (RHEED) system that can monitor 20 samples in parallel, and a time-of-flight ion scattering and recoil spectroscopy (TOF-ISARS) system for chemical analysis of the film surface. These advanced surface-science tools provide real time information about the film surface morphology, chemical composition, and crystal structure, and are quintessential for the success in growing atomically smooth and perfect films ${ }^{12}$. 
Using this system, we have performed over 2,500 LSCO film growth experiments so far. Each film was characterized in real time by RHEED and ex situ by atomic force microscopy (AFM) and magnetic susceptibility measurements. RHEED oscillations provide a digital count of atomic layers, and real-time control of film quality. Selected films were also characterized in situ by time-of-flight ion scattering and recoil spectroscopy and ex situ by X-ray diffraction (XRD), transport measurements, and Rutherford backscattering. Additional characterization was done by our collaborators using atomic-resolution scanning transmission electron microscopy and electron energy loss spectroscopy, resonant elastic and inelastic synchrotron X-ray scattering, synchrotron based X-ray phase-retrieval techniques such as coherent Bragg rod analysis, muon spin rotation, ultrafast electron diffraction, ultrafast optical and THz pump-probe techniques, etc.

Using ALL-MBE, we synthesize single-crystal thin films of LSCO that are atomically smooth, without any secondary phase precipitates or pinholes. The films can be made ultrathin, down to a single unit cell thick; this is advantageous for transmission measurements, because a relatively large transmittance helps minimize the effect of any small-area defects such as pinholes that can arise e.g., from imperfections in substrate polishing. Much thicker films are also readily grown for reflectance measurements.

To alleviate the problem of oxygen non-uniformity, we have performed over a thousand experiments that involved annealing in ozone, oxygen, or vacuum, spanning 13 orders of magnitude in pressure, from $10^{-8}$ Torr to $200 \mathrm{~atm}$. Before and after each annealing step, the films were characterized by AFM, transport, and XRD. Based on these extensive studies, we have developed recipes that involve multiple annealing steps at different temperature and pressure, yielding the most homogeneous films, with the sharpest superconducting transitions. 


\section{Atomic-layer engineering}

Uncertainty in the film thickness is another key problem that limits the accuracy of some measurements, e.g. of transmittance or the critical current density. ALL-MBE solves this problem by providing digital control over the film thickness - we count atomic monolayers, while we determine the lattice constant with crystallographic accuracy by X-ray diffraction. However, this still leaves some uncertainty about the actual thickness of the superfluid, because we have found that typically a couple of layers next to the substrate are modified structurally and chemically, and are not superconducting. The same is generally true for a couple of layers near the free film surface, once the film has been exposed to contamination from the atmosphere. To eliminate this problem, we resort to atomic-layer engineering.

An example is illustrated in Figure 1d. The active (superconducting) part of the sample under study is an exactly 5 unit cells (5 UC) thick HTS layer. It is protected on both sides by a metallic $\left(M=\mathrm{La}_{1.60} \mathrm{Sr}_{0.40} \mathrm{CuO}_{4}\right)$ buffer and a cover layer of fixed thickness. However, were it not for further sample engineering, there would be some hole depletion from $M$ and accumulation in the nearest HTS layers ${ }^{13}$. To minimize this interfacial effect, we 'sculpt' the charge profile near the interfaces by graded Sr doping in the four relevant layers. Moreover, we additionally dope these transition layers by substituting $3 \%$ of $\mathrm{Cu}$ with $\mathrm{Zn}$; this is $\mathrm{known}^{14}$ to suppress $T_{c}$ by a factor of 2 and $n_{s}$ even more dramatically, 4-5 times. In this way, we quench any potential residual superconductivity, and eliminate any interface contributions. One is then left with exactly $5 \mathrm{UC}$ of su-

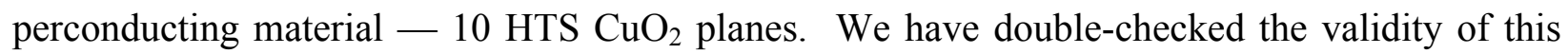
approach by synthesizing a series of hetero-structures in which we kept the composition of the constituent materials unaltered, but varying the thickness of the active HTS layers, and verifying 
that the sheet superfluid density scaled linearly with the number of $\mathrm{HTS}_{\mathrm{CuO}}$ planes (see Extended Data Fig. 1).

Note that this is just one example; indeed, we have synthesized a large number of samples, varying widely the layering scheme, the composition and thickness of individual layers, etc. The results presented in this paper are valid generally and not restricted to any of these choices.

\section{Penetration depth measurements}

Most frequently, $\lambda$ is measured using muon spin rotation $(\mu \mathrm{SR})^{4-8,31}$, microwave resonance (cavity-perturbation $)^{20-22,33-34}$, or mutual inductance ${ }^{9,10,15-17,35-52}$ techniques. Because of the cost and duration, $\mu \mathrm{SR}$ experiments are usually restricted to just a few compositions and temperatures. The microwave technique offers an unsurpassed relative accuracy but the absolute accuracy is limited by the uncertainty in geometric factors. For these reasons, and because it is best suited for thin film studies, we have chosen the mutual inductance method, pioneered by several groups ${ }^{10}$ ${ }^{15,16}$ (Figures 1a and 1b). A detailed theoretical treatment ${ }^{17}$ of a superconducting film of thickness $d$ and infinite radius, characterized by the ac conductivity $\sigma(\omega)=\sigma_{1}(\omega)-i \sigma_{2}(\omega)$, where $\omega$ is the measurement (angular) frequency, placed between two coils of radii $R_{1}$ and $R_{2}$, respectively, parallel to one another, and separated by a distance $D$, provided the following expression for the mutual inductance:

$$
\begin{aligned}
\hat{M}= & \operatorname{Re} M+i \operatorname{ImM}= \\
& \mu_{0} \pi R_{1} R_{2} \int_{0}^{\infty} d q\left[\exp (-q D) J_{l}\left(q R_{1}\right) J_{l}\left(q R_{2}\right)\right] /\left\{\cosh (Q d)+\left[\left(Q^{2}+q^{2}\right) / 2 q Q\right] \sinh (Q d)\right.
\end{aligned}
$$

where $\mu_{0}=4 \pi \times 10^{-7} \mathrm{~F} / \mathrm{m}, q$ is the wave-vector, $\mathrm{J}_{1}(x)$ is the first-order Bessel function, $Q^{2}=q^{2}+$ $\left(1 / \lambda^{2}\right)-i \mu_{0} \omega \sigma_{l}$, and $\sigma_{2}=1 / \mu_{0} \omega \lambda^{2}$. This is readily generalized to the case of two solenoids with 
$N_{1}$ and $N_{2}$ turns, respectively, by the summation over each pair of coils, one from each side. Once the values of $\operatorname{Re} M$ and $\operatorname{Im} M$ are measured experimentally, using eq. (1) one can determine the values of $\lambda$ and $\sigma_{1}$.

In practice, this procedure is subject to some uncertainties, because of imprecision in the coil geometry, run-to-run variations in the film position, the finite size of the film that allows some flux to 'leak' around it, other parasitic coupling between the two solenoids, and inaccuracy in the film thickness. In addition, the measurements are done down to some finite temperature, most frequently $T=4.2 \mathrm{~K}$, and the limiting value $\lambda_{0}$ is obtained by extrapolation. These uncertainties have been analyzed in detail in the literature, and various solutions were suggested how to reduce them. In what follows, we explain briefly the improvements we made in the measuring apparatus and technique in order to tighten the error bars.

Parasitic field coupling around the film and through the electronics. We use inductance coils with a large number of turns $(300-1,500)$ but very small inner radius $(250 \mu \mathrm{m})$, much smaller than the film size $\left(10 \times 10 \mathrm{~mm}^{2}\right)$, so that field leakage around the film is minimal $(<0.3 \%)$ to begin with. We nevertheless subtract it accurately based on measurements on films of $\mathrm{Nb}, \mathrm{Pb}$, and $\mathrm{Al}$, thick enough for transmittance to be negligible, and deposited on identical substrates. Alternatively, we deposit thick Al over-layer atop the HTS film, and switch the diamagnetic screening in $\mathrm{Al}$ (at $T=0.3 \mathrm{~K}$ ) between total (zero transmittance) and essentially none (total transmittance), by applying a small dc magnetic field (typically 100 Gauss) that drives Al normal but does not affect the HTS film. As a consistency check, we verified that the corrected $N_{s 0}$ scales linearly with the film thickness, while being independent on frequency. (In contrast, the leakage contribution varies with frequency, linearly for $10 \mathrm{kHz}<v<100 \mathrm{kHz}$, and thus can be clearly 
identified by repeating measurements on the same film at several frequencies.) To minimize eddy currents, we built the sample holder out of a single block of sapphire crystal. The coils are fixed rigidly and the sample is spring-loaded so that the film surface always gets to exactly the same position. Overall, we have achieved reproducibility and precision of $\pm 0.3 \%$, or better.

Uncertainty in coil geometry. Our measured mutual inductance $M(T)$ differs a little (typically by a couple per cent) from the calculated one, due to some deviations in the geometry of the actual coil from its idealized mathematical model. To account for this (multiplicative) factor, we normalize the measured $M(T)$ by its value $M_{\text {high }}$ just above $T_{c}$, or equivalently by $M_{s u b}$ of a pristine substrate (the later is essentially independent on temperature and equal to $M_{\text {high }}$ ).

Field penetration through secondary phase precipitates, scratches, and pinholes. This is particularly dangerous for thicker films where the intrinsic transmission is small. For this reason, we synthesize by ALL-MBE very thin films, down to $0.5 \mathrm{UC}$ thick. Also, we have a very large statistics, so we can easily recognize extrinsic behavior and factors.

Uncertainty in the film thickness. This is probably the single largest source of error ${ }^{37,43}$ in the measurements of $\lambda$ by inductance techniques reported so far. We have minimized this using digital synthesis as described in the Atomic-layer engineering section above, and illustrated in Figure 1d. We also compared films of the same composition and $T_{c}$ but of different thickness, as illustrated in Extended Data Fig. 1, and verified that the results are consistent. Altogether, this is the best that we can do today. Regrettably, the technique described in Fig. 1d would not work on the underdoped side, but that does not affect our main goal here, to study the evolution of LSCO physics from overdoped metal to the optimal doping. 
Extrapolation to $T \rightarrow 0$ of measurements done only down to $T=4 \mathrm{~K}$. While this has been done routinely in the literature, it may introduce error, especially for films with very low $T_{c}$ - say, below about $10 \mathrm{~K}$. Since we are interested in behavior near the quantum critical points where $T_{c}$ vanishes, we have built a $\mathrm{He}-3$ based setup extending the temperature range down to $300 \mathrm{mK}$. In the same setup, it is also possible to apply a dc magnetic field up to $9 \mathrm{~T}$. To ensure the accuracy in temperature reading, we measure upon both cooling and heating, at a very low rate of $0.1-0.2$ $\mathrm{K} / \mathrm{min}$, in a setup where we achieve $\mathrm{e}^{54}$ thermal stability better than $1 \mathrm{mK}$.

We measure $\lambda(T)$ with the reproducibility better than $\pm 0.3 \%$, as illustrated for a Nb film in Extended Data Fig. 2. Altogether, the accuracy in absolute value of $\lambda$ is better than $\pm 1 \%$. Moreover, some of our conclusions derive from the temperature dependence of $\lambda$, which is measured with the relative accuracy better than $\pm 0.1 \%$. The same is true of $\rho_{s}$, since $\rho_{s}=A / \lambda^{2}$, and $A=$ $\hbar^{2} d / 4 \mu_{0} k_{B} e^{2}=3.55 \times 10^{-12} \mathrm{~m}^{2} \mathrm{~K}$.

On selected films, we also compared our results to in-house high-frequency $(0.5-50 \mathrm{MHz}) \mathrm{in}-$ ductance measurements in the reflectance geometry ${ }^{51,52}$, as well as to the results of THz pumpprobe $^{55}$ and $\mu$ SR experiments ${ }^{31}$ performed by our collaborators, and found good agreement.

So far, we have performed inductance measurements on more than 2,500 LSCO films, a number of which were measured dozens of times and on multiple (a total of ten so far) setups. Mining this large database allows one to identify clear statistical trends and uncover intrinsic behavior. In this paper, we focus on the films with the sharpest transitions; in the best ones, near $T_{c}$ we see $\sigma_{I}(\omega, T)$ rising exponentially on the scale of $0.1-0.2 \mathrm{~K}$. This puts an upper bound on any inhomogeneity in $T_{c}$, since the transition width comes largely from thermal fluctuations. Clearly, these 
samples are very homogeneous, and hence they can be presumed to display intrinsic properties and behavior.

\section{The mobile carrier density}

Note that the values of $p$ quoted here, and in the literature, are only approximately equal to the mobile carrier (hole) density. A prevailing convention in the field is to infer $p$ from the measured $T_{c}$, assuming a parabolic $T_{c}(p)$ relation. This label $p$ should roughly indicate the doping state in a particular sample. To make it easier for the reader to compare our data with various phase diagrams in the literature, in Fig. 3 we provide the nominal $p$ values inferred by assuming that in $\operatorname{LSCO} T_{c}=A\left(p-p_{c 1}\right)\left(p_{c 2}-p\right)$, with $p_{c 1}=0.06, p_{c 2}=0.26$ and $A=4.15 \times 10^{3} \mathrm{~K}$, so that $p \equiv 0.16+$ $\left(0.01-2.4 \times 10^{-4} T_{c}\right)^{1 / 2}$. However, we stress that all our conclusions are in fact only based on the quantities such as $T_{c}, \lambda, \rho_{s}$, etc., that we measure directly and accurately.

$2,230 / 3,000$

\section{References}

31. Morenzoni, E., et al. The Meissner effect in a strongly underdoped cuprate above its critical temperature. Nature Commun. 2, 272 (2011).

32. Bonn, D. A. \& Hardy, W. N. Microwave surface impedance of high-temperature superconductors. In Physical Properties of High Temperature Superconductors V, edited by D. M. Ginsberg (World Scientific, Singapore, 1996) pp. 7-98.

33. Prozorov, R. \& Giannetta, R. W. Magnetic penetration depth in unconventional superconductors. Supercond. Sci. Tech. 19, R41-R67 (2006).

34. Prozorov, R. \& Kogan, V. G. London penetration depth in iron-based superconductors. Rep. Prog. Phys. 74, 124505 (2011). 
35. Lee, J. Y \& Lemberger, T. R. Penetration depth $\lambda(T)$ of $\mathrm{YBa}_{2} \mathrm{Cu}_{3} \mathrm{O}_{7-\delta}$ films determined from the kinetic inductance. Appl. Phys. Lett. 62, 2419-2421 (1993).

36. Pippard, A. B. Magnetic penetration depth through a superconducting film. Supercond, Sci. Tech. 7, 696-699 (1994).

37. Turneaure, S. J., Ulm, E. R. \& Lemberger, T. R. Numerical modeling of a two-coil apparatus for measuring the magnetic penetration depth in superconducting films and arrays. J. Appl. Phys. 79, 4221-4227 (1996).

38. Fuchs, A., Prusseit, W., Berberich, P. \& Kinder, H. High-precision penetration-depth measurement of $\mathrm{YBa}_{2} \mathrm{Cu}_{3} \mathrm{O}_{7-d}$ as a function of oxygen content. Phys. Rev. B. 53, 14745-14748 (1996).

39. Gilchrist, J. \& Brandt, E. H. Screening effect of Ohmic and superconducting planar thin films. Phys. Rev. B 54, 3530-3544 (1996).

40. Lee, J. Y., Kim, Y. H., Hahn, T-S. \& Choi, S. S. Determining the absolute value of penetration depth of large area films. Appl. Phys. Lett. 69, 1637-1639 (1996).

41. Locquet, J. P. et al. Variation of the in-plane penetration depth $\lambda_{a b}$ as a function of doping in $\operatorname{La}_{2}$ ${ }_{x} \mathrm{Sr}_{x} \mathrm{CuO}_{4+\delta}$ thin films on $\mathrm{SrTiO}_{3}$ : Implications for the overdoped state. Phys. Rev. B 54, 7481-7488 (1996).

42. Claassen, J. H., Wilson, M. L., Byers, J. M. \& Adrian, S. Optimizing the two-coil mutual inductance measurement of the superconducting penetration depth in thin films. J. Appl. Phys. 82, 3028-3034 (1997).

43. Turneaure, S. J., Pesetski, A. A. \& Lemberger, T. R. Numerical modeling and experimental considerations for a two-coil apparatus to measure the complex conductivity of superconducting films. J. Appl. Phys. 83, 4334-4343 (1998).

44. Paget, K. M., et al. Magnetic penetration depth in superconducting $\mathrm{La}_{2-x} \mathrm{Sr}_{x} \mathrm{CuO}_{4}$ films. Phys. Rev. B. 59, 641-646 (1999).

45. Wang, R. F., Zhao, S. P., Chen, G. H. \& Yang, Q. S. Absolute measurement of penetration depth in a superconducting film by the two-coil technique. Appl. Phys. Lett. 75, 3865-3867 (1999).

46. Coffey, M. W. Analyzing mutual inductance measurements to determine the London penetration depth. J. Appl. Phys. 87, 4344-4351 (2000).

47. Coffey, M. W. Mutual inductance of superconducting thin films. J. Appl. Phys. 89, 5570-5577 (2001). 
48. Rufenacht, A., Locquet, J. P., Fompeyrine, J., Caimi, D. \& Martinoli, P. Electrostatic modulation of the superfluid density in an ultrathin $\mathrm{La}_{2-x} \mathrm{Sr}_{x} \mathrm{CuO}_{4}$ film. Phys. Rev. Lett. 96, 227002 (2006).

49. Lemberger, T. R., Hetel, I., Tsukada, A. \& Naito, M. Anomalously sharp superconducting transitions in overdoped $\mathrm{La}_{2-x} \mathrm{Sr}_{x} \mathrm{CuO}_{4}$ films. Phys. Rev. B 82, 214513 (2010).

50. Lemberger, T. R., Hetel, I., Tsukada, A., Naito, M. \& Randeria, M. Superconductor-to-metal quantum phase transition in overdoped $\mathrm{La}_{2-x} \mathrm{Sr}_{x} \mathrm{CuO}_{4}$. Phys. Rev. B 83, 140507 (2011).

51. Gauzzi, A., et al. Very high resolution measurement of the penetration depth of superconductors by a novel single-coil inductance technique. Rev. Sci. Instrum. 71, 2147-2153 (2000).

52. Gasparov, V. A. \& Bozovic, I. Magnetic field and temperature dependence of complex conductance of ultrathin $\mathrm{La}_{1.55} \mathrm{Sr}_{0.45} \mathrm{CuO}_{4} / \mathrm{La}_{2} \mathrm{CuO}_{4}$ films. Phys. Rev. B 86, 094523 (2012).

53. Došlić, M., Pelc, D. \& Požek, M. Contactless measurement of nonlinear conductivity in the radiofrequency range. Rev. Sci. Instrum. 85, 073905 (2014).

54. Dubuis, G., He, X. \& Božović, I. Ultra-thermal-stabilization of a closed cycle cryocooler. Rev. Sci. Instrum. 85, 103902 (2014).

55. Bilbro, L. S. et al. Temporal correlations of superconductivity above the transition temperature in $\mathrm{La}_{2-x} \mathrm{Sr}_{x} \mathrm{CuO}_{4}$ probed by terahertz spectroscopy. Nat. Phys. 7, 298-302 (2011).

56. Donnelly, R. J. Cryogenics. In "Physics Vade Mecum", edited by H. L. Anderson (AIP New York 1981) p. 121, Table E.

57. Homes, C. C., Dordevic, S. V., Bonn, D. A., Liang, R. \& Hardy, W. N. Sum rules and energy scales in the high-temperature superconductor $\mathrm{YBa}_{2} \mathrm{Cu}_{3} \mathrm{O}_{6+x}$. Phys. Rev. B 69, 024514 (2004).

58. Homes, C. C., Dordevic, S. V., Valla, T. \& Strongin, M. Scaling of the superfluid density in hightemperature superconductors. Phys. Rev. B 72, 134517 (2005).

59. Tallon, J. L., Cooper, J. R., Naqib, S. H. \& Loram, J. W. Scaling relation for the superfluid density of cuprate superconductors: Origins and limits. Phys. Rev. B 73, 180504(R) (2006). 


\section{Extended Data}

\section{The demise of superfluid in overdoped copper oxide superconductors}

I. Božović, X. He, J. Wu \& A. T. Bollinger

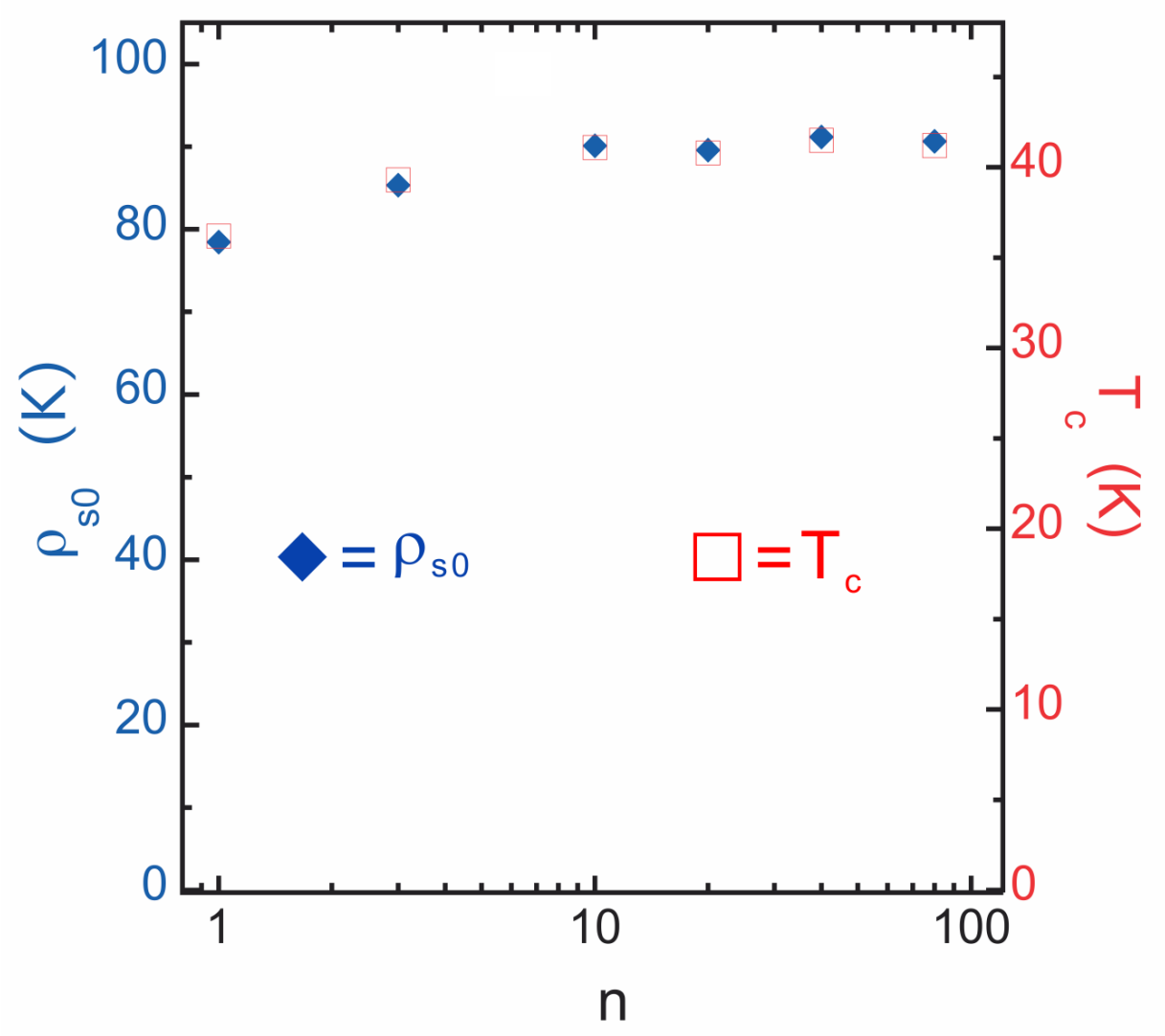

Figure $1 \mid$ The dependence of $T_{c}$ and $\rho_{s 0}$ in several LSCO films with the same nominal doping near the optimal ( $p=0.16$ ) in the active (superconductive) layer, but with different thickness $D=$ $n d$, where $d=0.662 \mathrm{~nm}$ and $n=1,2,4,10,40$, and 80, respectively. While individually both $T_{c}$ and $\rho_{s 0}$ show some random variations, in part due to imperfect control of the doping level and the density of oxygen vacancies, their ratio apparently stays virtually constant, to about $\pm 1 \%$. This reinforces the conclusion that $T_{c}$ is indeed essentially controlled by $\rho_{s 0}$, a purely kinematic quantity. 

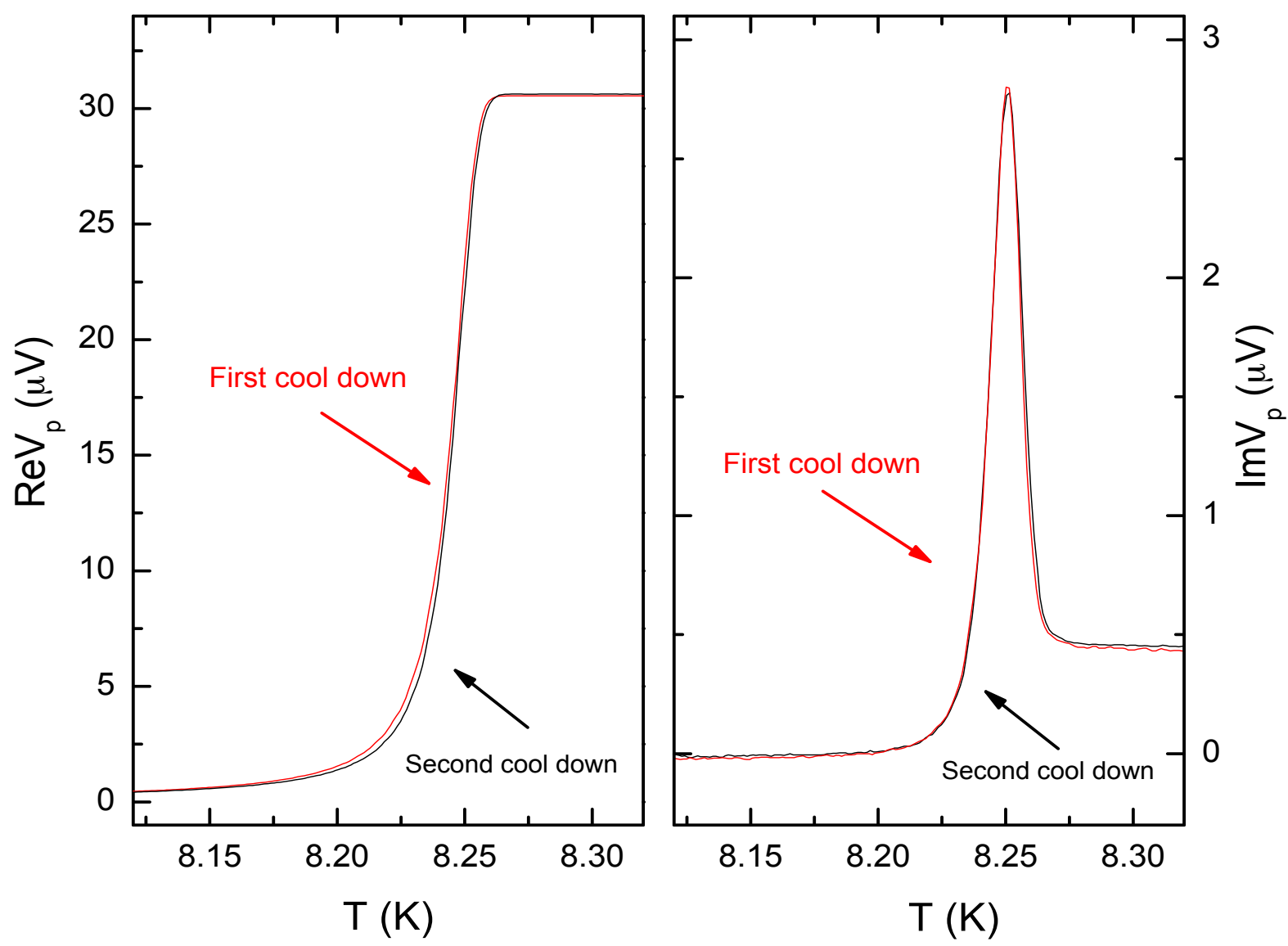

Figure 2 Mutual inductance (raw data) measured on a (275 \pm 12$) \mathrm{nm}$ thick $\mathrm{Nb}$ film deposited on standard $10 \times 10 \times 1 \mathrm{~mm}^{3} \mathrm{LaSrAlO}_{4}$ substrate on 03.22.2015 (red lines) and 01.20.2016 (black lines). The thermal stabilization is better than $\pm 1 \mathrm{mK}$ and the overall reproducibility better than $\pm 0.3 \%$, on one-year scale. The inferred value of $\lambda_{0}=(41 \pm 5) \mathrm{nm}$ agrees with the literature ${ }^{56}$; the error bar here largely comes from the uncertainty in the film thickness. This error is much smaller (down to less than $\pm 1 \%$ ) in the case of our LSCO films, where we employ atomic-layer deposition that provides digital control of the film thickness. 


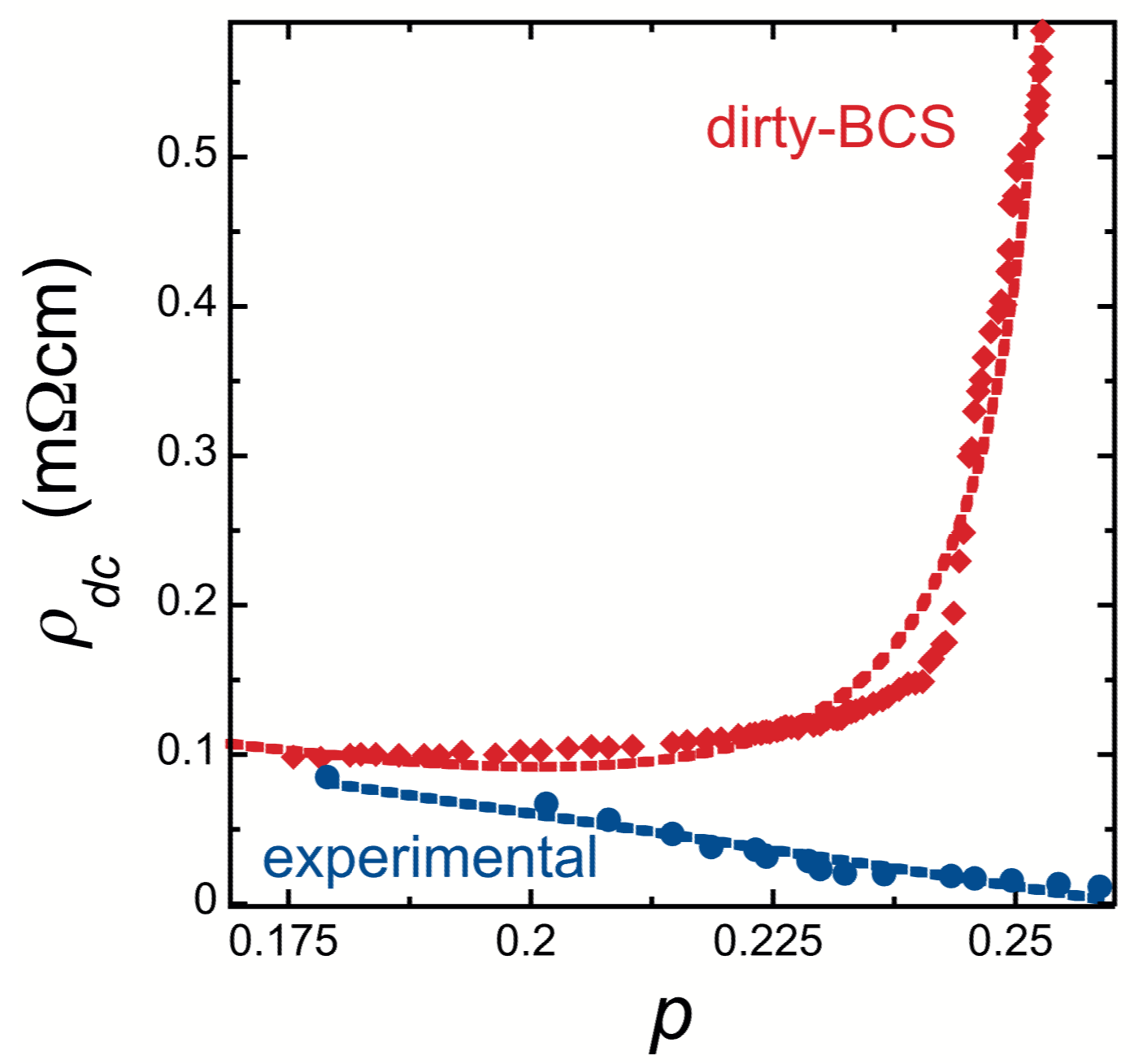

Figure 3 | Red diamonds: $\rho_{d c}$ calculated from our measured $T_{c}$ and $\rho_{s 0}$ values by applying Homes' Law, $\rho_{s 0} \propto \sigma_{d c} T$, that follows from the Ferrell-Glover-Tinkham sum rule for dirty BCS superconductors ${ }^{23,28,57-59}$. Since $T_{c} \propto \sqrt{ } \rho_{s 0}$ at high overdoping, the predicted $\rho_{d c}$ diverges as $1 / \sqrt{ } \rho_{s 0}$, which should trigger a superconductor-to-insulator transition. Red dashed line: a fit to $f(p)=$ $c_{1}+c_{2} p+c_{3} /(0.26-p)$. Blue circles: the measured $\rho_{d c}$ (from the data shown in Fig. 3b) showing that the samples in fact get more metallic. Blue dashed line: a fit to $f(p)=c_{1}-c_{2} p$. The gross discrepancy with the experiment implies that the original premise - the dirty-BCS scenario — is incorrect. 

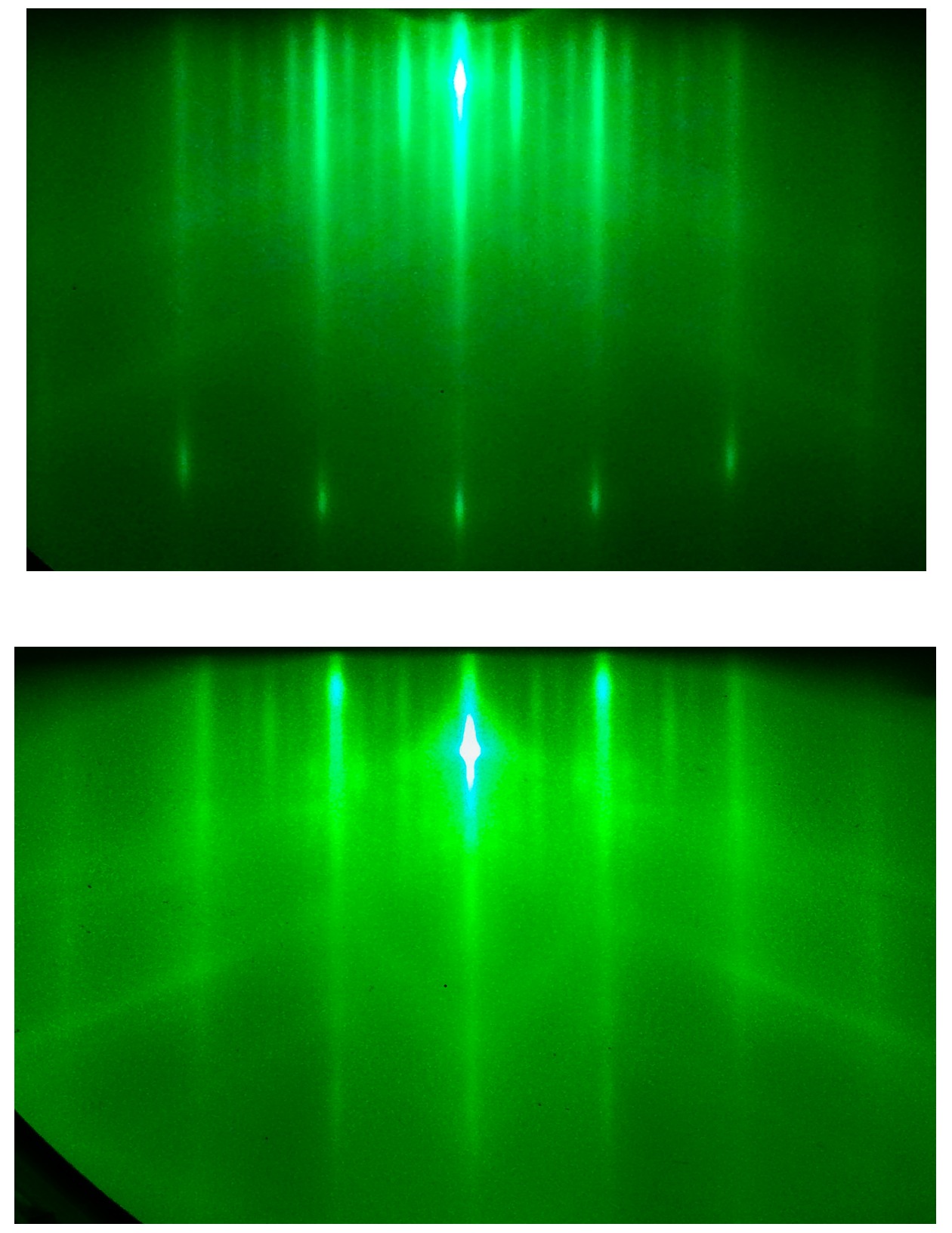

Figure 4 | RHEED recorded during growth of LSCO films by ALL-MBE. Top panel: an optimally doped ( $p=0.16, T_{c}=40 \mathrm{~K}$ ) LSCO film after the end of growth. Bottom panel: a strongly overdoped LSCO film ( $\left.p=0.24, T_{c}=7.5 \mathrm{~K}\right)$. The stronger, long main streaks correspond to Bragg-rod reflections at a very shallow angle from a terraced surface. The four weaker sidebands in between every pair of main streaks indicate an ubiquitous $5 \times 5$ surface reconstruction. The diagonal streaks are so-called Kikuchi lines formed in diffraction patterns by inelastically scattered electrons; they are observable only from atomically perfect surfaces. 


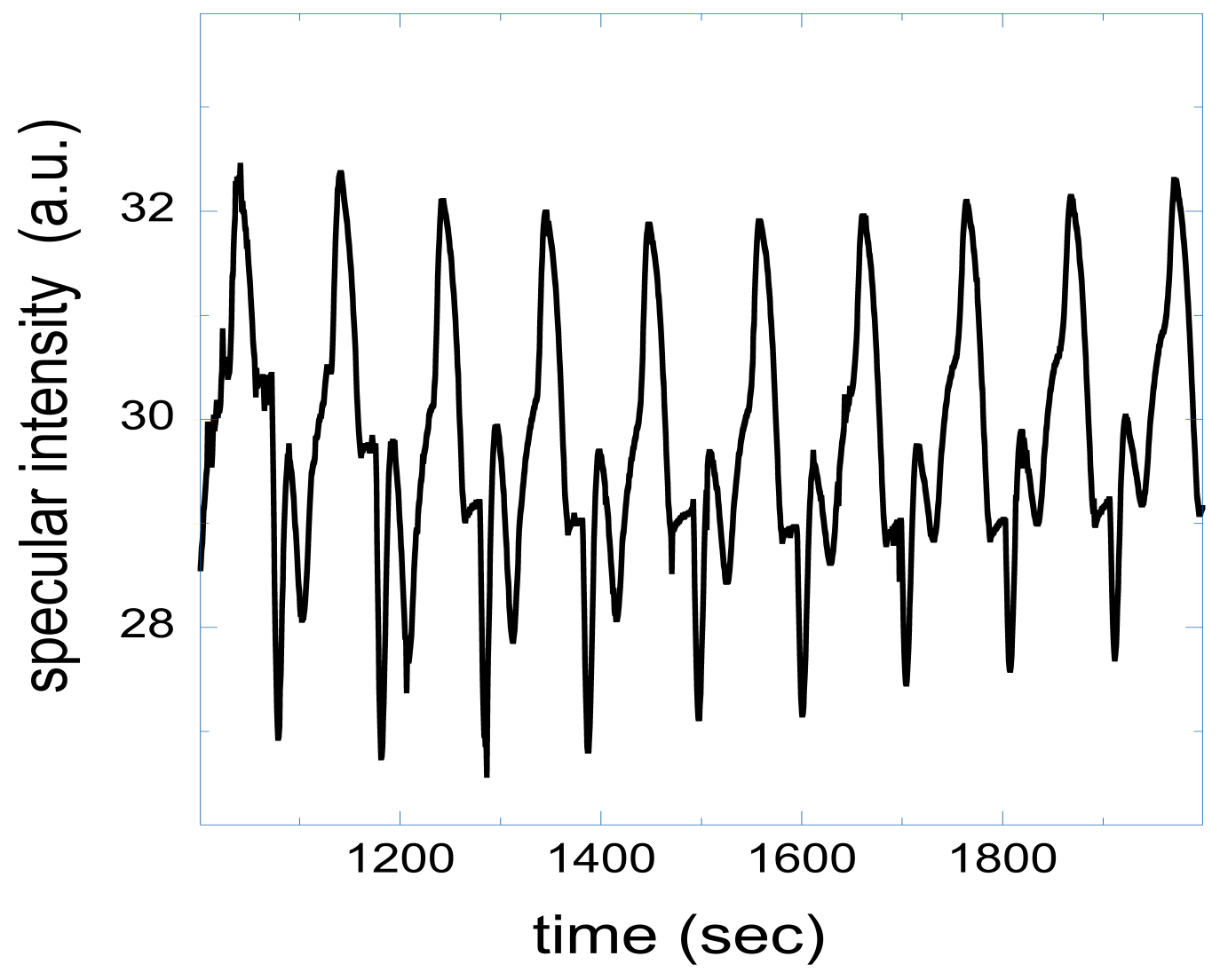

Figure 5 RHEED oscillations recorded during growth of LSCO film by ALL-MBE. In the atomic-layer growth mode, the intensity of the specular beam oscillates. When some 2D islands form on the surface, the diffuse reflectance increases as the specular reflectance decreases, until about one-half of the surface is covered. Then the specular reflectance increases again, reaching a new maximum at the full coverage. The fact that the amplitude of oscillations does not decrease indicates perfect atomic-layer growth. The number of periods provides digital information on the film thickness, expressed in the units of the lattice constant (which we know accurately from XRD). 


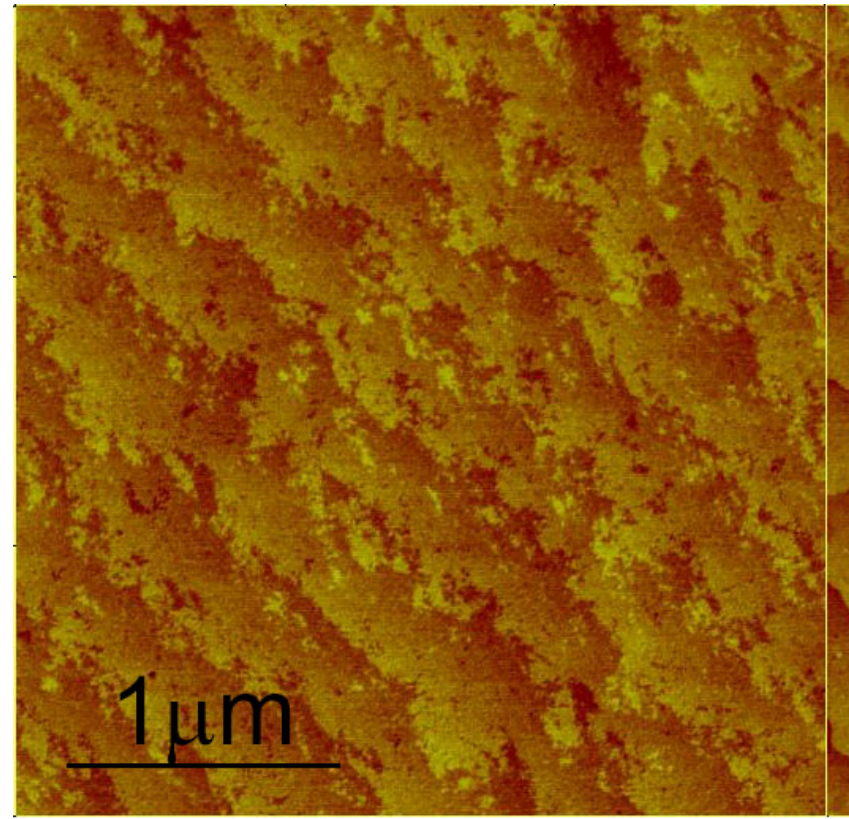

LSAO substrate rms roughness: $0.205 \mathrm{~nm}$

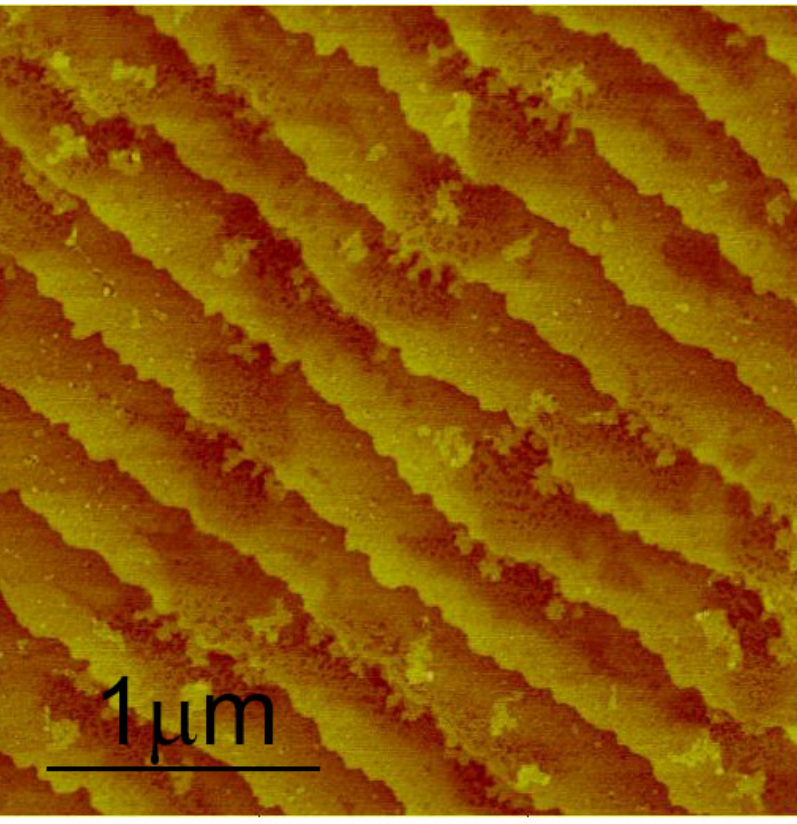

LSCO film rms roughness: $0.239 \mathrm{~nm}$

Figure 6| Atomic-force microscopy (AFM) images showing the quality of film surfaces. Left panel: an LSAO substrate. The steps, $0.5 \mathrm{UC}(0.65 \mathrm{~nm})$ tall, occur because the polished surface is unintentionally (but unavoidably) oriented slightly (typically by less than $0.3^{0}$ ) off the desired crystallographic plane perpendicular to the [001] direction. Right panel: a $225 \AA$ thick LSCO film grown on the same substrate. The steps in the substrate are projected onto the film and persist all the way to the film surface, indicating atomic-layer growth. The overall root-mean-square (rms) surface roughness is about $0.24 \mathrm{~nm}$; the terraces between steps are atomically smooth. 

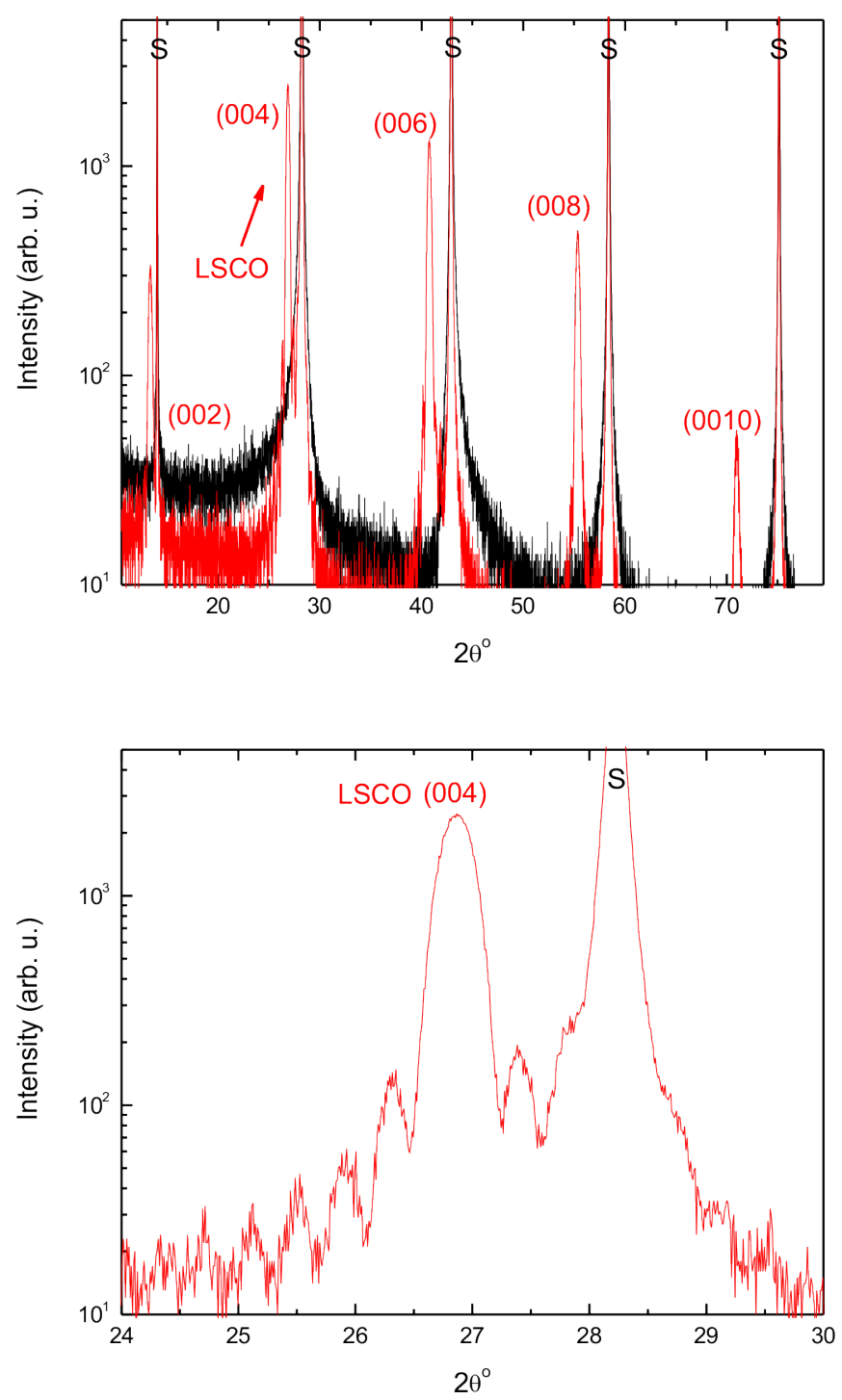

Figure 7 A wide angle $\Theta-2 \Theta$ X-ray diffraction (XRD) pattern of an LSCO film grown on an LSAO substrate by ALL-MBE. Upper panel: black curve, a pristine LSAO substrate; red curve, an LSCO film grown on the same substrate. Only even-order reflections are allowed by spacegroup symmetry. The substrate peaks are labeled with an ' $\mathrm{S}$ '. There are no traces of any other phases. Lower panel: an expanded view near the (004) LSCO reflection. The side-bands inbetween the LSCO Bragg reflections are the so-called Laüe (or 'finite-thickness') fringes that originate from interference between X-rays reflected from the film surface and the substrate-film interface. 


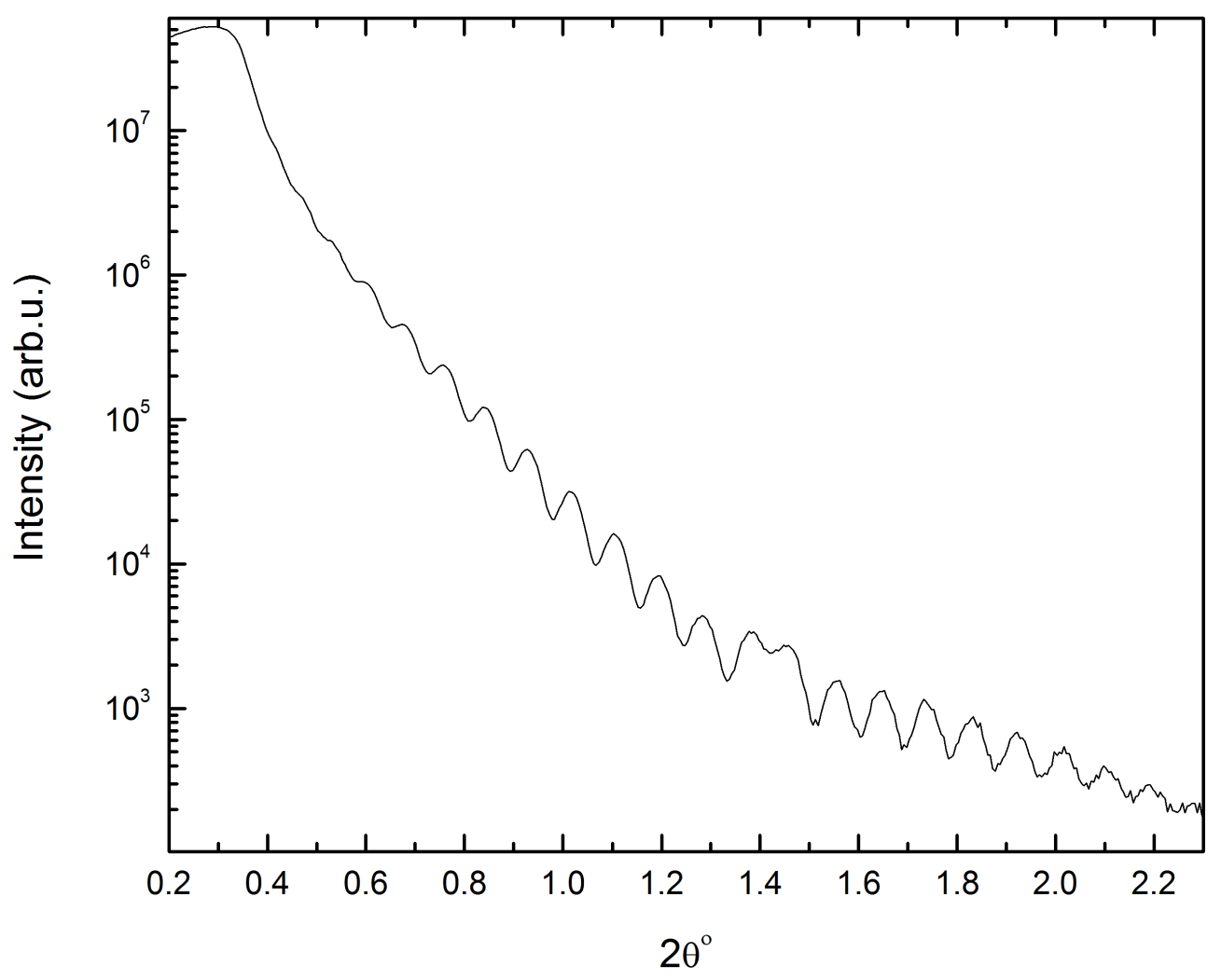

Figure 8 | Low-angle X-ray reflectivity (XRR) measured from an LSCO film grown on LSAO substrate by ALL-MBE. The oscillations are so-called Kiesig fringes that originate from interference between X-rays reflected from the film surface and the substrate-film interface. They are analogous to a Fabry-Perrot interferogram, and indicate that the two 'mirrors' are smooth and parallel on the scale of the wavelength of light (here, $1.54 \AA$ ). Comparing to simulated interferograms, one can estimate the film thickness and roughness; the estimates agree well with the thickness inferred from the digital count of unit cells by RHEED and the surface roughness determined by AFM. 